\title{
Risk associated with heparin withdrawal in ischaemic cerebrovascular disease
}

\author{
ANDREW SLIVKA, * DAVID E LEVY, ROBERT H LAPINSKI \\ From the Cerebrovascular Disease Research Center, Department of Neurology, Cornell University Medical \\ College, 1300 York Avenue, New York and Department of Neurology, ${ }^{*}$ Ohio State University, 1655 Upham \\ Drive, Columbus, Ohio, United States
}

SUMMARY Intravenous heparin is frequently used to treat thromboembolic disease, but the consequences of stopping heparin have not been studied systematically. To determine whether discontinuing heparin poses a clinical risk, we examined the charts of 378 patients treated with heparin for transient ischaemic attack (TIA), reversible ischaemic neurological deficit, or ischaemic stroke from October 1979 to June 1985. Clinical deterioration, or a new TIA or stroke was more likely $(\mathrm{p}=0.01)$ during the 24 hours after heparin was stopped in patients not already on aspirin or warfarin $(10 / 143,7 \%)$ than in patients receiving aspirin or warfarin before heparin withdrawal $(3 / 215,1 \%)$. Stopping heparin in patients not receiving aspirin or warfarin appears to expose them to an increased risk for TIA, stroke, or clinical deterioration.

Heparin is frequently used to treat ischaemic cerebrovascular disease. The complications of heparin therapy are well described and include haemorrhage, thrombocytopenia, which is rarely associated with arterial thrombosis, and osteoporosis. The consequences of terminating heparin therapy are not well known. Experimental studies have demonstrated thrombosis after heparin withdrawal. Although an intravenous heparin bolus inhibited thrombus formation in the extracorporeal circulation of pigs, compared to untreated controls, the tendency for thrombosis was enhanced 4-5 hours later. ${ }^{1}$ Similarly Ashwin ${ }^{2}$ found that rats had a higher incidence of jugular venous thrombosis four hours after an intravenous heparin injection than after intravenous saline. These experimental findings seem to have clinical relevance Alvarez Sabin $e \mathrm{al}^{3}$ described a patient with infarction of the internal capsule who subsequently developed a cerebellar infarct three days after stopping heparin. Systemic embolisation after inadvertent interruption of heparin treatment has been reported ${ }^{4}$ and we recently observed several patients on heparin for transient ischaemic attacks (TIA) who developed

Correspondence to: Dr A Slivka, Department of Neurology, Ohio State University, 1655 Upham Drive, Columbus, Ohio 43210, United States.

Received 13 January 1989 and in revised form 10 May 1989. Accepted 27 June 1989 cerebral infarcts soon after heparin was stopped. These experimental and clinical observations suggest that stopping heparin might predispose to thromboembolism and prompted us to examine our records systematically to determine the frequency of clinical deterioration following termination of heparin treatment.

\section{Methods}

At The New York Hospital data on hospital patients with transient ischaemic attacks (TIA), reversible ischaemic neurological deficits (RIND), and ischaemic stroke are entered into the Cornell Neurology Database. Patients with TIA/RIND exhibited focal neurological dysfunction not attributable to migraine or nonvascular disorders (for example, brain tumour, seizure disorder or trauma) that resolved before hospital discharge. Patients with ischaemic stroke had focal neurological dysfunction that persisted to the time of hospital discharge. Severe strokes were defined on the basis of the physical examination on admission as alteration of consciousness, hemiplegia in at least one limb and/or global aphasia.

Patients with TIA, RIND or stroke who were treated with heparin during the period October 1979 to June 1985, were identified from the Database. Hospital charts of these patients were then obtained and the following features noted: 1) age and sex; 2) diagnosis (TIA/RIND or stroke); 3) stroke severity; 4) duration of heparin treatment; 5) type of antithrombotic therapy, if any, received before and after heparin was discontinued; 6) clinical status while on heparin, after heparin was stopped and at discharge from the hospital; 7) 
admission platelet count, prothrombin time (PT) and partial thromboplastin time (PTT); 8) range of PTTs while on heparin, and if clinical deterioration occurred in a patient on heparin, the PTTs on that day; and 9) reasons for stopping heparin. Patients were considered to have completed a course of treatment if there was no apparent explanation from the chart for stopping heparin such as surgery, invasive procedure or treatment complication and if heparin was not restarted once stopped.

Heparin therapy was routinely given by continuous intravenous infusion with or without an initial bolus of 5,000 to 10,000 units. No patient remained on intravenous heparin indefinitely, and heparin was discontinued abruptly in nearly all patients. We divided patients into two groups based on whether they received other antithrombotic treatment before heparin was stopped. The first group of patients (noreplacement) did not receive warfarin or antiplatelet agents before heparin was stopped. In some of these patients antiplatelet agents were started several hours to days after heparin was stopped. In others, heparin therapy was subsequently reinstated, meaning that some patients had heparin started and stopped several times during their hospital stay. The second group of patients (replacement) was started on either warfarin or antiplatelet agents before their heparin treatment finished. Warfarin treatment typically started three to four days before heparin was discontinued and antiplatelet agents usually began several hours to one day before stopping heparin.

The end-point was a clinical event, categorised on the basis of the daily progress notes as 1) a new TIA, 2) a new ischaemic stroke, 3 ) acute deterioration of a stroke deficit which had been stable or improving for at least 24 hours, 4) progressive deterioration of a stroke deficit that was fluctuating and not stable for more than several hours at a time, or 5) venous thrombophlebitis. Each event was associated with at least one new or progressive physical abnormality. Events occurring during heparin treatment were tabulated for both replacement and non-replacement patients.

To investigate the hypothesis that stopping heparin without replacement increases the risk for an event, we determined the number of patients who had an event during the 24 hours before and after heparin was stopped in the replacement and no-replacement groups. A 1-tailed Fisher exact test was used for these analyses based on the hypothesis suggested by experimental studies and our anecdotal experience that stopping heparin without replacement increased the risk of an event. Twenty four hours was chosen as the time frame for comparison between the groups for several reasons. First, experimental evidence suggests the greatest risk of enhanced thrombosis occurs within six hours after stopping heparin. Also, adequate follow up information was not available on many patients for longer than 24 hours after stopping heparin. Replacement patients receiving antiplatelet agents and non-replacement patients were often discharged from the hospital 24 hours after heparin was stopped.

\section{Results}

In the 68 months of the study period, 401 patients were given heparin for TIA/RIND or ischaemic stroke. Some TIA patients received heparin because they had multiple attacks. The majority of patients with RIND or stroke who were given heparin had either a cardiac source of emboli, a partial deficit or progressive signs. We excluded 23 patients from the analysis because the relevant portions of their charts could not be located. We have no reason to suspect that the excluded patients have clinical features that differ from the patients included in the study. Of the 378 remaining patients, 216 were in the replacement group (183 received warfarin and 33 antiplatelet agents) and 162 were in the no-replacement group. All patients had CT soon after admission, and none of these initial scans demonstrated haemorrhage or mass lesions.

The replacement and no replacement groups were similar in age, sex, diagnosis and stroke severity (table 1). Replacement patients, however, were treated significantly longer (median 7 days, range 1-43 days) than no-replacement patients (median 4 days, range 0.5-19 days; $p<0.01$, Wilcoxon). This discrepancy probably reflects the reasons for stopping heparin in the two groups. Heparin was discontinued in all but seven replacement patients because the treatment course was considered complete, whereas this was true of only one third of no-replacement patients (table 2). Heparin was stopped in most of the remaining patients before a procedure or because of treatment complications. The percentage of patients with an event on heparin was comparable in the non-replacement (34/ $145,23 \%)$ and replacement groups $(42 / 215,20 \%)$. We excluded eighteen patients (17 in the no-replacement group and 1 in the replacement group) with potential nonthrombotic explanations for events on heparin including cerebral haemorrhage, seizures, hypotension, bradycardia, syncope, endarectomy, or respiratory arrest.

A striking feature about the events during heparin

Table 1 Comparison of replacement and no-replacement patients

Replacement No-replacement

\begin{tabular}{|c|c|c|c|}
\hline $\begin{array}{l}\text { Number of patients } \\
\text { Age: Mean } \\
\text { Range } \\
\text { Sex (male:female) }\end{array}$ & $\begin{array}{l}216 \\
66 \\
28-89 \\
141: 75\end{array}$ & $\begin{array}{l}162 \\
66 \\
22-91 \\
91: 71\end{array}$ & $\begin{array}{l}\text { NS } \\
\text { NS }\end{array}$ \\
\hline $\begin{array}{l}\text { Diagnostic category TIA/RIND: } \\
\text { Ischaemic stroke }\end{array}$ & & & \\
\hline $\begin{array}{l}\text { Number of patients with severe } \\
\text { stroke }\end{array}$ & .1. & 14 & NS \\
\hline $\begin{array}{l}\text { Time on heparin before discontin } \\
\text { Median } \\
\text { Interquartile range }\end{array}$ & $\begin{array}{c}\text { ation (day } \\
7 \\
4-11\end{array}$ & $\begin{array}{l}4 \\
1-8\end{array}$ & * \\
\hline $\begin{array}{l}\text { Number of patients with events } \\
\text { while on heparin }\end{array}$ & $42 / 215 \dagger$ & $34 / 145 \dagger$ & NS \\
\hline
\end{tabular}

NS Not significant (2-tailed chi square test except age, which was evaluated with the Wilcoxon test).

*p $<0.01$ (Wilcoxon test).

†Eighteen patients (17 in the no-replacement group and 1 in the replacement group) with potential nonthrombotic explanations for events on heparin were excluded. 
Table 2 Reasons for stopping heparin in the no-replacement group

Number of patients

Number of times heparin stopped

Reasons for stopping heparin:

Heparin course completed

Bleeding*

Angiography

Endarterectomy

Unknown

Miscellaneous $\dagger$

Invasive procedure

No improvement or severe deficit

162

198

71

29

27

15

15

13

*Cerebral haemorrhage occurred in 11 of these 29 patients.

†Miscellaneous reasons included considerably elevated PTT, possible endocarditis, clinical worsening questionably related to intracranial haemorrhage, and difficulty maintaining intravenous access. Heparin was stopped in two patients because of a marked fall in the platelet count; neither had events on heparin or after it was stopped.

treatment in both no-replacement and replacement patients was their timing. The median time from the onset of heparin therapy until an event was two days, with a range of 0.5 to 25 days. Of patients with events on heparin, $82 \%(62 / 76)$ had an event within the first two days of treatment, and $92 \%(70 / 76)$ had one within four days of starting heparin. Events were thus concentrated in the first several days after starting treatment.

Events during heparin treatment cannot be explained by inadequate anticoagulation (measured by the PTT) since nearly all of the patients with nonhaemorrhagic events on heparin had concurrent PTT values at least 1.5 times the control value. In three patients no pertinent laboratory values were available. Although five ischaemic stroke patients had a PTT value less than 1.5 times the control value at some point while worsening, most of their values during deterioration were greater than 1.5 times the control value.

After heparin was discontinued, 10 of 143 patients $(7 \%)$ in the no-replacement group had a total of 14 events (table 3). All 10 patients had cerebral events, TIA, new ischaemic stroke, or acute deterioration of an existing deficit, and one (case 10) also developed venous thrombophlebitis. All five non-replacement patients with strokes who had events after heparin withdrawal were clinically stable or improving before the heparin was withdrawn and none were admitted with severe strokes. After heparin was stopped these patients experienced an acute deterioration of their deficit which in all cases subsequently improved over the next 24 hours. Twelve of the 14 events occurred within 24 hours of stopping heparin (median $7 \cdot 5$ hours). Both of the events occurring later than 24 hours were in patients (cases 2,3 ) who also had an event within one day of stopping heparin. Moreover, of four patients in whom heparin was started and stopped several times during their hospital course (cases $2,3,7,10$ ), three had an event each time heparin was stopped (cases $2,3,7$ ).

These 10 no-replacement patients with events after heparin withdrawal were similar to the remainder of the no-replacement population. Their mean age was 64 years (six women and four men). Only two patients (cases 4,5 ) or $20 \%$ also had events (TIAs) while on heparin. This proportion is comparable to the $23 \%$ $(34 / 145)$ of patients in the no-replacement group as a whole who had events while on heparin. On the other $\bigcirc$ hand, heparin was not stopped in any of these $10 \AA$ patients because the heparin treatment course was complete, even though this was the most common reason for stopping heparin therapy in the noreplacement group (table 2). Also, $50 \%$ (5/10) of patients in this group were admitted with TIAs compared to $30 \%(48 / 162)$ in the no-replacement group as a whole. More patients in the no-replacement group had events in the 24 hours after stopping heparin $(10 / 143,7 \%)$ than in the replacement group $(3 / 215,1 \%, p=0.01$, table 4). Significantly more

Table 3 Details of events after stopping heparin in the no-replacement group

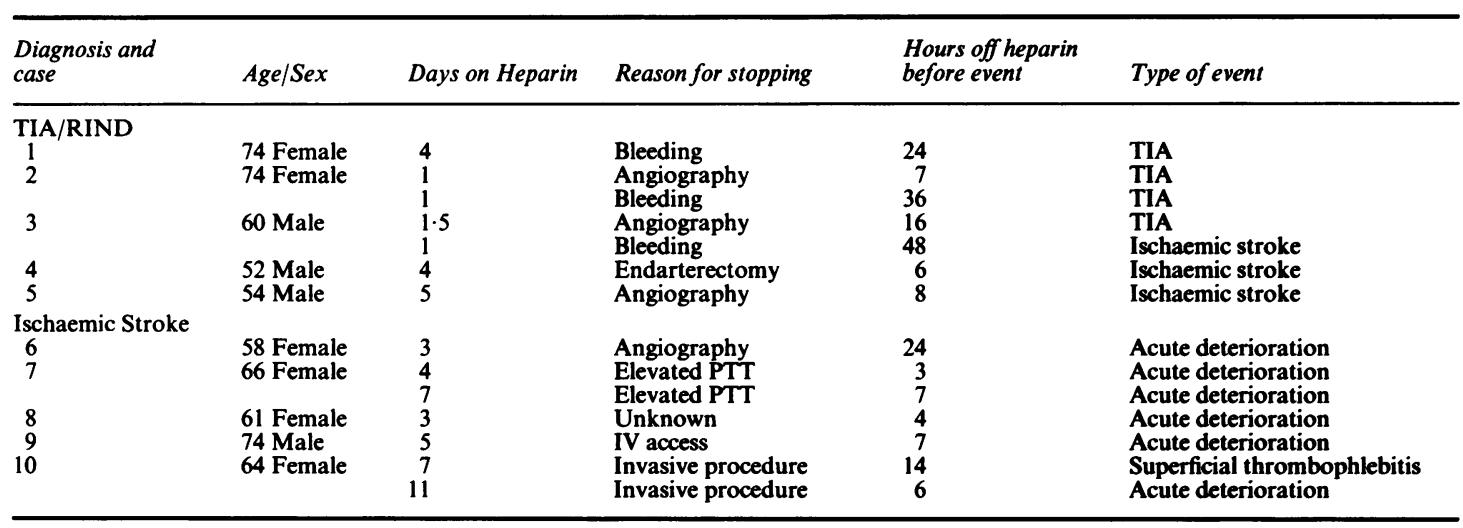


Table 4 Risk of events in no-replacement and replacement groups

\begin{tabular}{lccc}
\hline & $\begin{array}{c}\text { 24 Hours before } \\
\text { stopping heparin }\end{array}$ & $\begin{array}{l}\text { 24 Hours after } \\
\text { stopping heparin }\end{array}$ \\
\hline $\begin{array}{l}\text { No-replacement } \\
\text { Event }\end{array}$ & 3 & 10 & \\
$\begin{array}{c}\text { No event } \\
\text { Replacement }\end{array}$ & 140 & 133 & $\mathrm{p}=0.04$ \\
$\begin{array}{l}\text { Event } \\
\text { No event }\end{array}$ & 33 & $3 \dagger$ & NS \\
& NS & 212 & \\
\hline
\end{tabular}

*Nineteen patients with potential explanations for events after stopping heparin were excluded from this group.

†Two of these patients were on warfarin and one was receiving an antiplatelet agent. One patient who had a TIA following cerebral angiography was excluded. Even if this patient is included the difference between the no-replacement and replacement groups remains significant, $p<0.05$. NS Not significant, Fisher exact test.

events occurred in the no-replacement group 24 hours after stopping heparin $(10 / 143,7 \%)$ than in the previous 24 hour $(3 / 143,2 \%, p=0.04)$. Nineteen patients in the no-replacement group who had events after stopping heparin were omitted from the above analyses because they had potential explanations for the events such as cerebral haemorrhage, endarterectomy, herniation, and encephalopathy following coronary artery surgery.

\section{Discussion}

Several potential limitations of our study need to be considered. One concern is its retrospective nature. While prospective, controlled trials are better suited than retrospective analyses to assess cause and effect relationships, they are not always practical. A prospective design of this study would require half the patients to stop receiving further antithrombotic therapy after their course of heparin was completed. This is unacceptable medical practice as in many patients continued anticoagulation with warfarin is indicated. Even if a prospective trial is ethically feasible, another consideration would be whether the potential clinical significance justifies the time and expense of the study. In this case, it would be difficult to justify a prospective study of sufficient size to determine whether stopping heparin without replacement antithrombotic therapy predisposes patients to further events if only $7 \%$ of such patients experience these events. For these reasons, we do not believe a prospective study to examine the risk of stopping heparin will be carried out.

Selection bias is a potential source of error in any retrospective study. Clearly we could not identify all the factors that could be important in influencing thrombotic events. In terms of the conclusions, however, the issue is not whether these variables were identified and matched between the two groups but whether for whatever reason one group was more susceptible to thrombotic events. If, for example, noreplacement patients were more unstable than replacement patients, they would be more likely to have events under any circumstance than replacement patients. Our results, however, do not demonstrate a difference in the clinical condition of the two groups. When the percentage of patients who had an event on heparin was analysed as an objective measure of clinical stability, the numbers were similar for replacement patients $(20 \%)$, no-replacement patients $(23 \%)$ and no-replacement patients with events after stopping heparin $(20 \%)$. A further consideration is that the difference in the length of therapy between the two groups may be a confounding variable. Since events occurring during heparin treatment are concentrated in the first four days, one might expect that noreplacement patients who were treated for a shorter duration (four days) than replacement patients (seven days) might be more likely to have an event whether heparin was stopped or not. Alternatively, a longer duration of heparin therapy may somehow be protective, in which case replacement patients would be expected to have fewer events than no-replacement patients, since they were treated for longer. To address this problem we analysed the number of events occurring 24 hours before stopping heparin in both no-replacement and replacement patients and found no statistical difference. However, we found significantly more events 24 hours after heparin was stopped compared to the previous 24 hours on heparin in no-replacement patients. There was no such difference in replacement patients. These results suggest that the difference in duration of heparin treatment between the two groups did not affect the results. Therefore, although the groups may not be perfectly comparable, our results do not suggest that noreplacement patients are more susceptible to events than replacement patients.

The most plausible cause for the events that occur after stopping heparin is that the thrombotic tendency which is held in check during heparin treatment is allowed to continue when the drug is stopped. Alternatively the underlying cerebral thrombotic process may actually be facilitated by stopping heparin. Stopping heparin, experimentally, has been associated with an enhanced tendency for thrombosis. ${ }^{34}$ Possible mechanisms for potentiation of thrombosis after heparin withdrawal include decreased antithrombin III or elevated factor VIII, both of which have been associated with thromboembolic disease $e^{5-10}$ and reported to occur during heparin therapy." 12

While the clinical events we measured (TIA, stroke or worsening of a stroke deficit) are frequently associated with thromboembolic processes, all can occur by other mechanisms. We excluded patients, 
most of them in the no-replacement group, with possible non-thrombotic explanations for events such as hypotension, herniation, arrhythmia, or seizures. The events occurring after heparin withdrawal in the five stroke patients (table 3 ) are not readily explained by cerebral oedema. None of the five patients had severe strokes, which are often associated with clinically significant oedema. Available evidence also suggests that cerebral oedema is maximal two to four days after infarction. ${ }^{13}$ Only two of the five patients experienced deterioration within four days after stroke onset and both occurred at the upper limit of this range on day four. Furthermore, all five of these patients were clinically stable or improving before the withdrawal of heparin, subsequently experienced an acute deterioration of their deficit and then improved over the next 24 hours. This clinical course is not characteristic of that seen with cerebral oedema. We therefore believe the events recorded in table 3 approximate the number of events related to thromboembolic processes.

In conclusion, stopping heparin in patients with TIA/RIND or ischaemic stroke without first beginning warfarin or antiplatelet agents may expose them to an increased risk of experiencing a clinical event. Even though clinical evidence of thromboembolism after heparin withdrawal occurred in only $7 \%$ of patients, our data suggest that starting warfarin or antiplatelet agents before interrupting a heparin course decreases this frequency.

We thank Drs Babette Weksler, John Kissel and Jerry Mendell for their helpful suggestions and Ms Abigail Sarokin and Tibitha Harris for preparation of the manuscript, Dr A Slivka received the NIH Training Grant No NS-07141 during this research study. This research was supported in part by NIH Grant No NS03346, the Robert Wood Johnson Foundation and the American Heart Association with funds supplied in part by the New York Heart Association.

\section{References}

1 Mustard JF, Murphy EA, Downie HG, Rowsell HC. Heparin and thrombus formation: early suppression and late enhancement Brit J Haemat 1963;9:548-51.

2 Ashwin JG. Elevated incidence of experimental thrombosis after heparin. Canad J Biochem Physiol 1962;40:1153-7.

3 Alvarez Sabin J, Matias-Guiu J, Sumalla J, Cordina Puiggros A, Neuvo infarto cerebral tras la retirada de anticoagulantes en una paciente. Med Clin (Bar) 1984;83:777-8.

4 Zinn WJ. Side reactions of heparin in clinical practice. Am J Cardiol 1964;14:36-8.

5 Egeberg $O$. Inherited antithrombin deficiency causing thrombophilia. Thromb Diath Haemorrh 1965;13:516-30.

6 Marciniak E, Farley CH, De Simone PA. Familial thrombosis due to antithrombin III deficiency. Blood 1974;43:219-31.

7 Mackie M, Bennett B, Ogston D, Douglas AS. Familial thrombosis: Inherited deficiency of antithrombin III. $\mathrm{Br} \mathrm{Med} \mathrm{J}$ 1978;1:136-8.

8 Cosgriff TM, Bishop DT, Hershgold EJ, et al. Familial antithrombin III deficiency: Its natural history, genetics, diagnosis, and treatment. Medicine 1983;62:209-20.

9 Penick GD, Dejanov II, Reddick EL, Roberts HR. Predisposition to intravascular coagulation. Thromb Diath Haemorrh (Suppl) 1966;21:543-4.

10 Owen CA, Bowie EJW, Didisheim P, et al. Factor VIII and TGA $\varrho$ types of hypercoagulability. In: Sherry S, Brinkhous KM Genton E, Stengle JM, eds. Thrombosis. Washington DC: National Academy Sciences, 1969:573.

11 Marciniak E, Gockerman JP. Heparin-induced decrease in cir culating antithrombin III. Lancet 1977; ii:581-4.

12 Denson DWE, Redman CWG. Heparin-induced decrease in circulating antithrombin III. Lancet 1977;2:1028-9.

13 Katzman R, Clasen R, Klatzo I, Meyer JS, Pappius HM, Waltz AG. IV. Brain edema in stroke. Stroke 1977;8:512-40. 